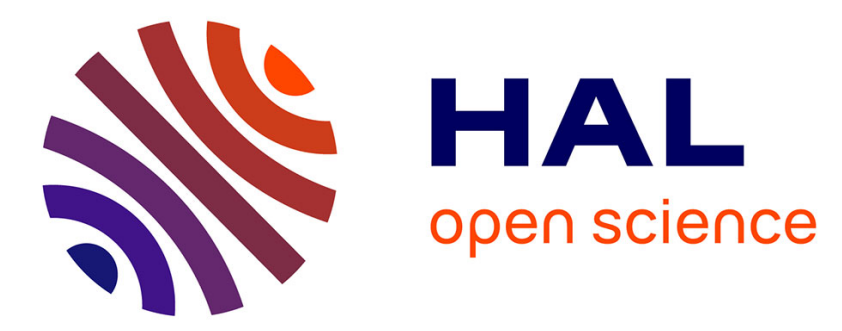

\title{
Photo-assisted inkjet printing of antibodies onto cellulose for the eco?-friendly preparation of immunoassay membranes
}

\author{
Julie Credou, Rita Faddoul, Thomas Berthelot
}

\section{To cite this version:}

Julie Credou, Rita Faddoul, Thomas Berthelot. Photo-assisted inkjet printing of antibodies onto cellulose for the eco?-friendly preparation of immunoassay membranes. RSC Advances, 2015, 5, pp.2978629798. 10.1039/C5RA03442F . hal-01156569

\author{
HAL Id: hal-01156569 \\ https://hal.science/hal-01156569
}

Submitted on 17 Nov 2015

HAL is a multi-disciplinary open access archive for the deposit and dissemination of scientific research documents, whether they are published or not. The documents may come from teaching and research institutions in France or abroad, or from public or private research centers.
L'archive ouverte pluridisciplinaire HAL, est destinée au dépôt et à la diffusion de documents scientifiques de niveau recherche, publiés ou non, émanant des établissements d'enseignement et de recherche français ou étrangers, des laboratoires publics ou privés. 


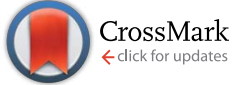

Cite this: RSC Adv., 2015, 5, 29786

Received 25th February 2015

Accepted 17th March 2015

DOI: $10.1039 / c 5 r a 03442 f$

www.rsc.org/advances

\section{Photo-assisted inkjet printing of antibodies onto cellulose for the $\mathrm{eco}^{2}$-friendly preparation of immunoassay membranes}

\begin{abstract}
Julie Credou, Rita Faddoul and Thomas Berthelot*
The current global issues have stimulated the search for both ecologically and economically friendly (eco ${ }^{2}$ friendly) materials and processes. As an affordable biopolymer, cellulose is an ideal material for developing diagnostic devices. Recently, paper-based bioanalytical devices have trended towards three-dimensional microfluidic platforms allowing multiplex diagnosis. This technological development now challenges the production process of those devices. In this perspective, the biomolecule immobilization process presented here combines an inkjet printing dispensing method with a photolinker-free photografting procedure. While many printing cycles are usually achieved to get efficient immune answers, only one to five printing passes were sufficient in this study, thereby enabling to save bio-inks. Antibodies have been successfully printed and immobilized onto paper sheets. These membranes were further used to perform lateral flow immunoassays. The visual detection limits observed were identical to those usually displayed by the classical dispensing method, regardless of the membrane material. Thus, the process developed herein is simple, time and cost-saving as well as environmentally friendly. More generally, it is a powerful tool for robust and abundant immobilization of chemical-sensitive proteins onto various cellulose-based papers and according to complex designs.
\end{abstract}

\section{Introduction}

The current ecological and economic global issues have result in an increasing desire for sustainable technologic development. Hence, the search for renewable-resources-based procedures and environmentally friendly materials and processes, as well as cost-saving approaches, has been stimulated widely. ${ }^{1}$

As the main component of plant skeleton, cellulose is an almost inexhaustible raw material ${ }^{2}$ and the most abundant form of worldwide biomass (about $1.5 \times 10^{12}$ tons per year). ${ }^{3}$ It is therefore an affordable biopolymer with lots of appealing properties such as large bioavailability, good biodegradability and biocompatibility. ${ }^{2,4}$ Moreover, cellulose is insoluble in most usual organic solvents. It swells but does not dissolve in water, hence enabling aqueous fluids and their contained components to penetrate within the fibers matrix and to wick by capillarity with no need for any external power source. With special regard to cellulose paper, porosity combined to biocompatibility allows biological compounds to be stored in the paper device. ${ }^{5}$ Besides, cellulose sheets are available in a broad range of thicknesses and well-defined pore sizes, easy to store and handle, and lastly safely disposable. ${ }^{6,7}$ All of its features make cellulose an ideal structural engineering material and a grade one platform for

CEA Saclay, IRAMIS, NIMBE, LICSEN (Laboratory of Innovation in Surface Chemistry and Nanosciences), F-91191 Gif sur Yvette, France. E-mail: julie.credou@cea.fr; rita. faddoul@cea.fr; thomas.berthelot@cea.fr; Fax:+33 169084044; Tel: +33 169086588 creating novel devices for diagnostics, microfluidics, and electronics. ${ }^{4}$ Thus, a new technological sector has risen within the last ten years: paper-based technology. ${ }^{8}$ Though paper-based immunoassay such as dipstick tests or lateral flow immunoassays (LFIAs) have been marketed and extensively employed for point-of-care (POC) diagnostics and pathogen detection since the $80 \mathrm{~s}$ (diabetes and pregnancy tests being the most famous), ${ }^{9-13}$ the recent impetus given to paper-based microfluidics by American, Canadian and Finnish research teams ${ }^{14-16}$ has resulted in the development of new paper-based bioanalytical devices with complex designs allowing multiplex diagnosis. ${ }^{17-20}$

Equipped with this sustainable, low-cost and easy to use material, the next challenge now lies in developing a production process as $\mathrm{eco}^{2}$-friendly as possible. Two parts of the process should therefore be considered: the whole device design and shaping on one hand, and the biosensing material dispensing and immobilization on the other hand.

Regarding the device shaping, the frame material of a multiplex device needs to be patterned with microfluidic channels. ${ }^{1}$ Thus, several methods for patterning paper sheets have been developed. ${ }^{18,21}$ Among the many processes are photolithography, using SU-8 or SC photoresist,, ,14,22 "wax printing" or "wax dipping", ${ }^{23-25}$ inkjet printing ${ }^{26}$ and laser cutting. ${ }^{27,28}$

With regard to the biosensing material, the spatially controlled immobilization of biomolecules is a key step in the 
development of biosensing devices. ${ }^{29}$ Photolithographic methods can be used to control protein immobilization onto selected specific areas of the substrate. Yet, this is a long and complex process composed of many steps. First of all, a photoresist is prepared and deposited onto the substrate through a master form. After UV exposure, the non-exposed regions are developed by chemical treatment. Biomolecules are finally immobilized on the non-developed regions. ${ }^{30,31}$ In addition to complexity, and resulting high cost, there is a not insignificant risk for biomolecules to come across traces of the toxic reagents and solvents used in the development step. This is why printing techniques such as micro-contact printing or inkjet printing are often preferred to spatially control biomolecule immobilization. ${ }^{32-35}$ Compared to photolithography, printing techniques allow quick cycles where only one step printing biomolecule - is required. Moreover, printing is considered a biocompatible environmentally friendly process. It is a versatile technique enabling the deposition of variable kinds of solutions (biomolecules, polymers, solvents, metals) onto different types of substrates (cellulose, polymer, glass, silicon) and according to any design desired. ${ }^{36,37}$ It is a fast dispensing process allowing low-cost, high throughput fabrication, ${ }^{37}$ and therefore a very attractive approach regarding the economic and ecological goals. However, to be able to detect an immune answer, many printing cycles were needed so far. For example, referring to Abe et al. works, 60 print cycles of an immune-sensing ink were necessary to detect $10 \mu \mathrm{g} \mathrm{L} \mathrm{L}^{-1}$ (i.e. 10 $\mathrm{ng} \mathrm{mL}^{-1}$ ) of IgG molecule ${ }^{34}$ and 24 cycles of protein ink were inkjet printed in order to detect $0.8 \mu \mathrm{M}$ of human serum albumin (HSA) (i.e. $53.6 \mu \mathrm{g} \mathrm{mL}{ }^{-1}$ since $M_{\mathrm{HSA}}=67 \mathrm{kDa}$ ). ${ }^{38}$ Moreover, printing is only a dispensing technique and is not sufficient by itself to strongly immobilize biomolecules onto cellulose. Recent findings revealed that about $40 \%$ of antibody molecules adsorbed onto cellulose paper can actually desorb from the fibers. ${ }^{39}$ Direct adsorption of antibodies onto cellulose is therefore too weak to allow the permanent immobilization required in the development of immunoassay. ${ }^{40}$ Cellulose activation or functionalization is thus necessary.

In the present study, printing parameters (jetting voltage and printing resolution) were controlled in order to allow low detection limits ( 1 to $25 \mathrm{ng} \mathrm{mL}^{-1}$ ) with only 1 and 5 printing passes. Furthermore, this process combines inkjet printing of biomolecules with a photolinker-free photografting procedure previously patented ${ }^{41-44}$ which ensures easy, rapid and strong immobilization of antibodies onto cellulose-based papers. Hence, the new process developed and presented herein not only is faster and more cost-saving than the known printing processes implemented in the development of paper-based biosensing devices, but also ensures a strong and precisely localized immobilization of antibodies onto paper. To put the process to the test, a simple lateral flow immunoassay (LFIA) device was first produced and studied. The model antigen used in these assays was ovalbumin (OVA) and the antibodies directed against its epitopes were murine monoclonal antibodies (mAbs). Each prepared membrane was subjected to several immunoassays. The first one evaluated the immobilization rate thanks to a gold-labeled goat anti-mouse tracer antibody. The other ones assessed the biological activity and evaluated the visual detection limit thanks to a gold labeled murine anti-OVA tracer antibody and OVA dilution series ranging from $0 \mathrm{ng} \mathrm{mL} L^{-1}$ (negative control) to $500 \mathrm{ng} \mathrm{mL}^{-1}$ (positive control). Every experiment was conducted in triplicate. Since adsorption on nitrocellulose is the most frequently used method for immunochromatographic assays, ${ }^{10,45}$ all results were analyzed with respect to nitrocellulose as the reference material. Likewise, the inkjet printing process was compared to the classical automatic dispensing method with BioDot-like systems usually implemented in LFIA preparation. ${ }^{46}$ Several parameters of the inkjet printing procedure have thus been optimized, as well as paper substrate pretreatment, therefore resulting in visual detection limits (VDLs) that challenge nitrocellulose values.

\section{Experimental}

\subsection{Reagents and reaction materials}

Proteins (ovalbumin (OVA), Bovine Serum Albumin (BSA) and porcine skin gelatin), as well as chemical products for preparing buffers, colloidal gold solution, and substrates pretreatment mixtures were obtained from Sigma-Aldrich (St Louis, MO, USA). Water used in all experiments was purified by the Milli-Q system (Millipore, Brussels, Belgium). Monoclonal murine antibodies (murine mAbs) were produced at LERI (CEA, Saclay, France) as previously described. ${ }^{47}$ Goat anti-mouse antibodies $(\operatorname{IgG}+\operatorname{IgM}(\mathrm{H}+\mathrm{L}))$ were purchased from Jackson ImmunoResearch (West Grove, PA, USA).

Papers used for preparing the immunoassay membranes were CF1 cellulose and AE 98 Fast nitrocellulose from Whatman (Maidstone, Kent, UK). Immunochromatographic strips were prepared using Standard 14 sample wick from Whatman (Maidstone, Kent, UK), no. 470 absorbent pad from Schleicher and Schuell BioScience GmBH (Dassel, Germany) and MIBA-020 backing card from Diagnostic Consulting Network (Carlsbad, CA, USA).

Antibody solutions were either printed onto substrates using a laboratory piezoelectric drop-on-demand inkjet printer Dimatix Materials Printer DMP-2831 (Fujifilm, Santa Clara, CA, USA) with $10 \mathrm{pL}$ nominal drop volume cartridge, or dispensed at $1 \mu \mathrm{L} \mathrm{cm}^{-1}$ using an automatic dispenser (XYZ3050 configured with 2 BioJet Quanti Dispenser (BioDot, Irvine, CA, USA)). Irradiations were conducted at room temperature in a UV chamber CN-15. LV UV viewing cabinet (Vilber Lourmat, Marne-la-Vallée, France). Strips were cut using an automatic programmable cutter Guillotine Cutting CM4000 Batch cutting system from BioDot (Irvine, CA, USA). 96-Well polystyrene microplates (flatbottom, crystal-clear, from Greiner Bio-One S.A.S. Division Bioscience, Les Ulis, France) were used as container for migrations on immunochromatographic strips. Opaque plastic (double-sided tape) maskings used in the photo-patterning experiments have been designed and prepared with a laser plotter LaserPro Spirit (GCC Laser Pro, New Taipei City, Taiwan), and the software CorelDRAW Graphics Suite (Corel Corporation, Ottawa, Canada). 


\subsection{Characterization materials}

Infrared (IR) spectra of the various substrates were recorded on a Vertex 70 FT-IR spectrometer (Bruker, Billerica, MA, USA) controlled by OPUS software (Bruker, Billerica, MA, USA) and fitted with MIRacle ${ }^{\mathrm{TM}}$ ATR (Attenuated Total Reflectance) sampling accessory (PIKE Technologies, Madison, WI, USA). The ATR crystal type was single reflection diamond/ZnSe crystal plate. The FT-IR detector was MCT working at liquid nitrogen temperature. Acquisitions were obtained at $2 \mathrm{~cm}^{-1}$ resolution after 256 scans.

X-ray photoelectron spectroscopy (XPS) studies of membranes were performed with an Axis Ultra DLD spectrometer (Kratos, Manchester, UK), using monochromatic $\mathrm{Al} \mathrm{K}_{\alpha}$ radiation $(1486.6 \mathrm{eV})$ at $150 \mathrm{~W}$ and a $90^{\circ}$ electron take-off angle. The area illuminated by the irradiation was about $2 \mathrm{~mm}$ in diameter. Survey scans were recorded with $1 \mathrm{eV}$ step and $160 \mathrm{eV}$ analyzer pass energy and the high-resolution regions with 0.05 $\mathrm{eV}$ step and $40 \mathrm{eV}$ analyzer pass energy. During the data acquisition, the sample surfaces were neutralized with slow thermal electrons emitted from a hot $\mathrm{W}$ filament and trapped above the sample by the magnetic field of the lens system (hybrid configuration). Referring to Johansson and Campbell's work, XPS analysis was carried out on dry samples, together with an in situ reference. ${ }^{48}$

Microstructure and surface morphology of samples were examined by a JSM-5510LV (JEOL, Tokyo, Japan) scanning electron microscope (SEM) after gold coating (K575X Turbo Sputter Coater (Quorum Technologies Ltd, Ashford, Kent, UK), working at $15 \mathrm{~mA}$ for 20 seconds). The images were acquired at various magnifications ranging from $100 \times$ to $3000 \times$. The acceleration voltage and working distance were $4 \mathrm{kV}$ and 17 $\mathrm{mm}$, respectively. Images were acquired applying the secondary electron detector.

Surface roughness, $R_{\mathrm{a}}$, of the unprinted substrates was measured with an AlphaStep ${ }^{\circledR}$ D-120 Stylus Profiler (KLATencor, Milpitas, CA, USA). Measurements were performed along a line of $1 \mathrm{~mm}$ long, with a stylus force of $1 \mathrm{mg}$ and a speed of $0.05 \mathrm{~mm} \mathrm{~s}^{-1}$.

Printed solutions viscosity was measured before printing with a MCR 102 Rheometer (Anton Paar, Ashland, VA, USA). Cone-plane geometry was used at a shear rate varying from 100 to $10000 \mathrm{~s}^{-1}$ and at a $24{ }^{\circ} \mathrm{C}$ temperature. Gap distance was equal to $0.1 \mathrm{~mm}$. Geometry diameter and angle were equal to 5 $\mathrm{cm}$ and $1^{\circ}$, respectively.

Colorimetric intensity resulting from colloidal gold on immunochromatographic strips was qualitatively estimated directly by eye at first and then indirectly through a picture taken with a Molecular Imager VersaDoc ${ }^{\mathrm{TM}}$ MP4000, in association with Quantity One 1-D Analysis software (Bio-Rad, Hercules, CA, USA). Colorimetric intensity resulting from colloidal gold on masked papers was quantified with the same imager and software.

\subsection{Substrates pretreatment}

AE 98 Fast nitrocellulose and CF1 cellulose were used as received. In addition, several pieces of CF1 cellulose were treated in order to obtain cellulose sheets enriched with glucose (glucose-cellulose) or paraffin (paraffin-cellulose). Glucosecellulose was prepared by dipping a CF1 cellulose sheet in a 100 $\mathrm{mg} \mathrm{mL} \mathrm{m}^{-1}$ aqueous solution of $\mathrm{d}(+)$-glucose overnight at $4{ }^{\circ} \mathrm{C}$, and then drying it at $37{ }^{\circ} \mathrm{C}$ in a ventilated oven for 1 hour. Similarly, paraffin-cellulose was prepared by dipping a cellulose sheet in a $10 \mathrm{mg} \mathrm{mL}^{-1}$ hot aqueous suspension of paraffin for 1 hour, and then drying it at $37^{\circ} \mathrm{C}$ in a ventilated oven for 1 hour. The temperature of the aqueous solution needed to be above 60 ${ }^{\circ} \mathrm{C}$ for paraffin to melt and mix with water.

\subsection{Immobilization procedure}

2.4.1. Printing. Antibody solutions were printed onto the raw and pretreated substrates using the Dimatix inkjet printer. Nozzle diameter was $21.5 \mu \mathrm{m}$ and nominal drop volume was 10 pL. Printing tests were performed at $40 \mathrm{~V}$ tension with $15 \mu \mathrm{m}$ drop spacing. While drop spacing is inversely proportionate to resolution, printing voltage is directly related to the ejected volume. The printed pattern (Fig. 1) consisted of two straight lines of $600 \mu \mathrm{m}$ width and was designed according to usual LFIA strips. ${ }^{46}$ The bottom line was therefore dedicated to capture the OVA model antigen (test line). The top line aimed to detect antiOVA tracer antibodies (control line). Thus, the test line consisted of murine anti-OVA monoclonal antibodies $\left(1 \mathrm{mg} \mathrm{mL} \mathrm{m}^{-1}\right.$ in $0.1 \mathrm{M}$ potassium phosphate buffer, $\mathrm{pH} 7.4$ ) and the control line of goat anti-mouse polyclonal antibodies $\left(0.5 \mathrm{mg} \mathrm{mL}^{-1}\right.$ in $0.1 \mathrm{M}$ potassium phosphate buffer, $\mathrm{pH}$ 7.4). Printings made of 1 and 5 layers were compared to the usual automatic dispensing method $\left(1 \mu \mathrm{L} \mathrm{cm}^{-1}\right.$ with the BioDot system $) .{ }^{46}$

2.4.2. Immobilization. Two procedures were implemented depending on the nature of the substrate. Thus, antibodies were adsorbed onto nitrocellulose substrate (AE 98 Fast nitrocellulose), while they were photoimmobilized onto cellulose substrates (CF1 cellulose, glucose-cellulose and paraffincellulose). Results obtained onto the raw and pretreated cellulose substrates were analyzed with respect to nitrocellulose as the reference material.

According to previous optimization results, ${ }^{\mathbf{4 1 , 4 4}}$ the photoimmobilization process for antibody immobilization onto cellulose can be described as follows: (i) an antibody solution was dispensed onto a cellulose sheet (see previous section); (ii) antibodies were concentrated by drying of the impregnated paper at $37{ }^{\circ} \mathrm{C}$, in a ventilated oven, for 15 minutes; (iii) the system was irradiated at $365 \mathrm{~nm}\left(1050 \mu \mathrm{W} \mathrm{cm}{ }^{-2}\right)$ for $2 \mathrm{~h} 40 \mathrm{~min}$ (about $10 \mathrm{~J} \mathrm{~cm}^{-2}$ ) for inducing photoimmobilization; and (iv) papers were intensively rinsed with a washing buffer $(0.1 \mathrm{M}$ potassium phosphate buffer, $\mathrm{pH} 7.4$, containing $0.5 \mathrm{M} \mathrm{NaCl}$ and $0.5 \%(\mathrm{v} / \mathrm{v})$ Tween 20$)$ for removing non-immobilized antibodies.

Adsorption of antibodies onto nitrocellulose was achieved by regular 1 hour incubation at room temperature and following washing step.

\subsection{Immunochromatographic assays (LFIA)}

Immobilization rate, biological activity and visual detection limit (VDL) of the antibody-printed membranes were evaluated by colloidal-gold-based lateral flow immunoassays (LFIAs). ${ }^{\mathbf{1 0}}$ The 
Control line

\begin{tabular}{lr} 
Control line & $0.6 \mathrm{~mm}$ \\
\hline Test line & $\sqrt[16 \mathrm{~mm}]{1 \mathrm{~cm}}$
\end{tabular}

Fig. 1 Scheme of the printed pattern.

(a)

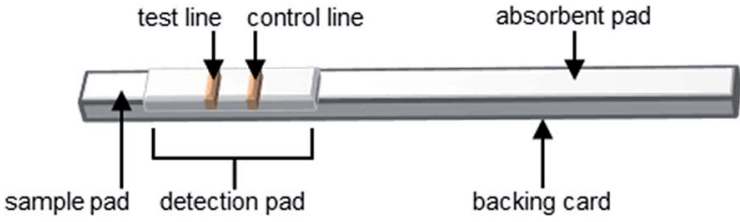

(b)

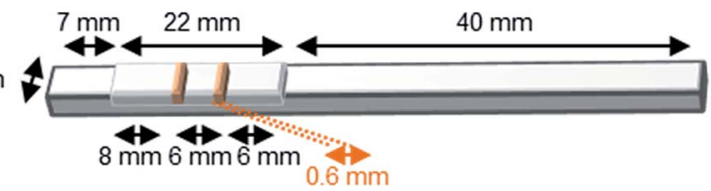

Fig. 2 Schematic representation (a) and proportioning (b) of an immunochromatographic strip.

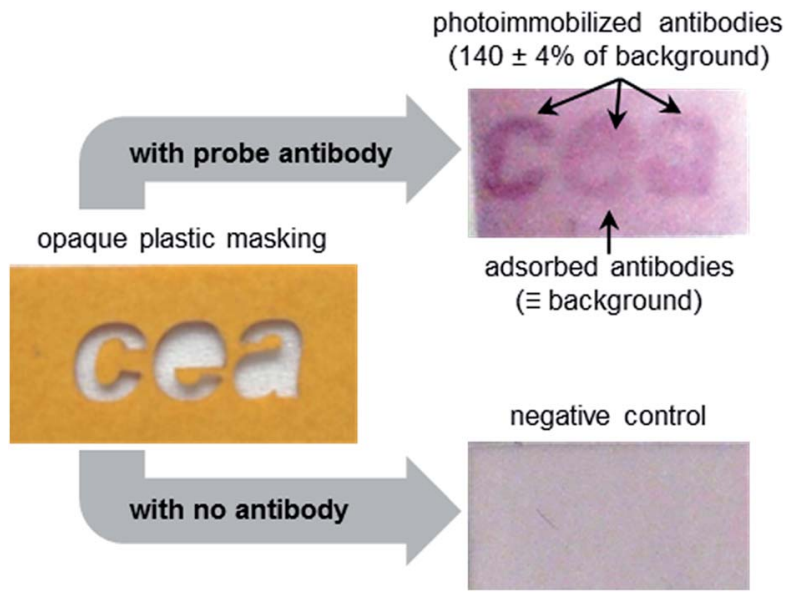

Fig. 3 Photo-patterning of gold-labeled goat anti-mouse tracer antibodies. Photographs were taken with a regular digital camera.

signal intensity was qualitatively estimated directly by eye at first and then indirectly through a picture taken with a Molecular Imager. All results were compared with those obtained with nitrocellulose which is the reference material.

All the reagents were diluted in the analysis buffer $(0.1 \mathrm{M}$ potassium phosphate buffer, $\mathrm{pH} 7.4$, containing $0.1 \%$ (w/v) BSA, $0.15 \mathrm{M} \mathrm{NaCl}$, and $0.5 \%(\mathrm{v} / \mathrm{v})$ Tween 20$)$, at room temperature, 30 minutes prior to migration in order to reduce nonspecific binding. Each assay was performed at room temperature by inserting a strip into a well of a 96-well microtiter plate containing $100 \mu \mathrm{L}$ of the test solution. The mixture was successively absorbed by the various pads and the capillary migration process lasted for about 15 minutes. Colorimetric intensity was immediately estimated by eye and pictures with both regular digital camera and Molecular Imager were taken without delay.
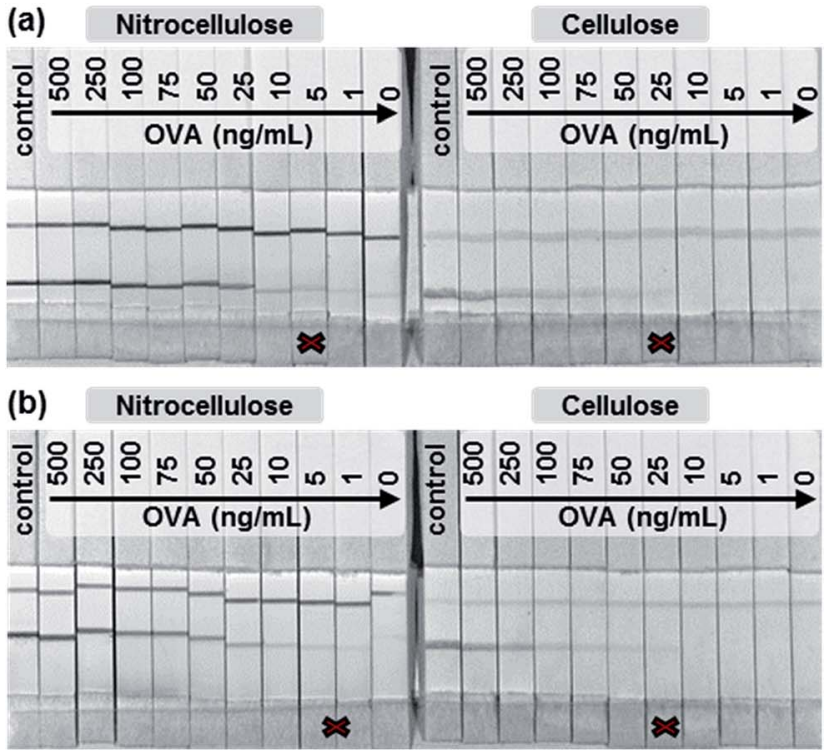

(c) Nitrocellulose

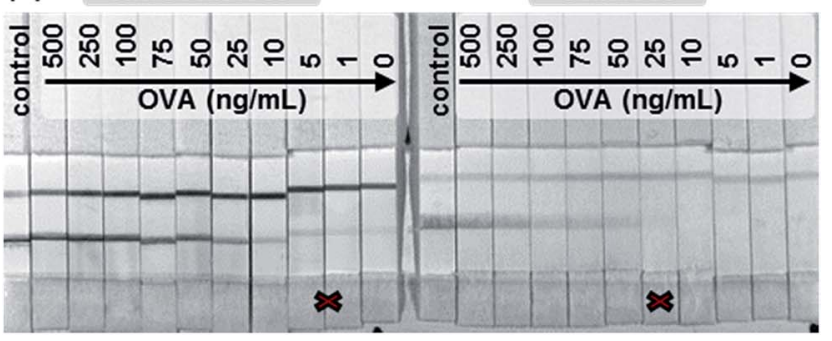

Fig. 4 Photographs showing the influence of the dispensing process on biological activity and membrane VDL. The first set of strips (a) results from usual BioDot dispensing method, the second (b) from 1layer inkjet printing, and the third (c) from 5-layer inkjet printing. Antibodies were adsorbed onto nitrocellulose and photoimmobilized onto cellulose. Their actual immobilization was confirmed thanks to gold-labeled goat anti-mouse tracer (control strips). The capture of OVA antigen by the immobilized antibodies was highlighted by goldlabeled murine anti-OVA tracer (OVA strips). The strips corresponding to the membranes' VDL are labeled with a cross. Photographs were taken with the Molecular Imager. All experiments were reproduced 3 times but only one is shown here.

2.5.1. Preparation of colloidal-gold-labeled antibodies. Tracer antibodies were labeled with colloidal gold according to a known method previously described. ${ }^{46}$ Two types of tracer were prepared: a goat anti-mouse tracer to reveal the immobilized murine antibodies, and a murine anti-OVA tracer to highlight the capture of OVA by the immobilized antibodies.

Briefly, $4 \mathrm{~mL}$ of gold chloride and $1 \mathrm{~mL}$ of $1 \%(\mathrm{w} / \mathrm{v})$ sodium citrate solution were added to $40 \mathrm{~mL}$ of boiling water under 

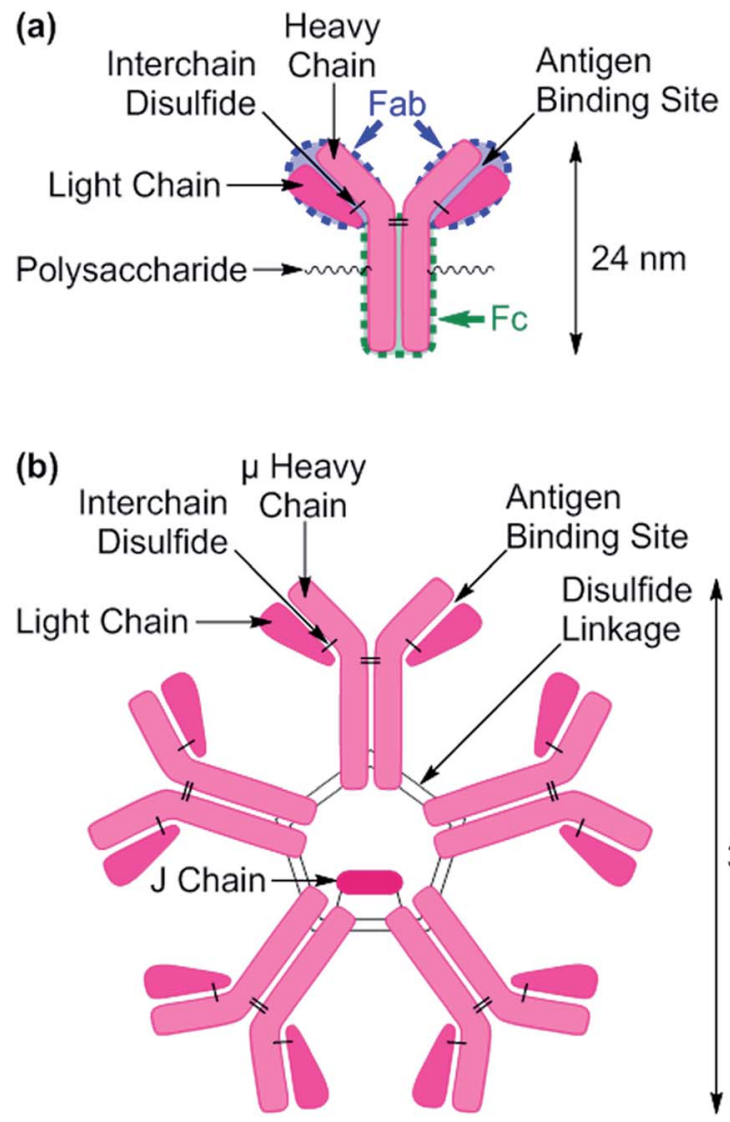

Fig. 5 Detailed structure of an IgG antibody molecule (a) and general structure of an IgM antibody molecule (b).

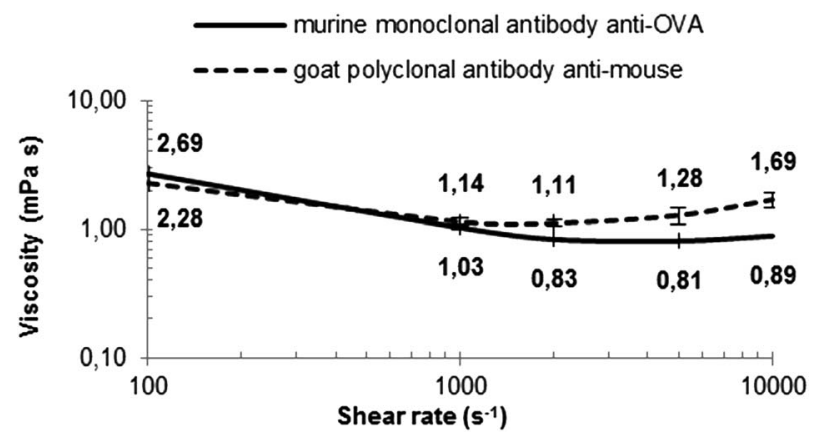

Fig. 6 Antibody solutions viscosities at $24^{\circ} \mathrm{C}$ and shear rate varying from 100 to $10000 \mathrm{~s}^{-1}$.

constant stirring. Once the mixture had turned purple, this colloidal gold solution was allowed to cool down to room temperature and stored at $4{ }^{\circ} \mathrm{C}$ in the dark. $25 \mu \mathrm{g}$ of $\mathrm{mAb}$ and 100 $\mu \mathrm{L}$ of $20 \mathrm{mM}$ borax buffer, $\mathrm{pH} 9.3$, were added to $1 \mathrm{~mL}$ of this colloidal gold solution. This mixture was left to incubate for one hour on a rotary shaker at room temperature, therefore enabling the ionic adsorption of the antibodies onto the surface of the colloidal gold particles. Afterwards, $100 \mu \mathrm{L}$ of $20 \mathrm{mM}$ borax buffer, $\mathrm{pH}$ 9.3, containing $1 \%(\mathrm{w} / \mathrm{v}) \mathrm{BSA}$, was added and the mixture was centrifuged at $15000 \mathrm{~g}$ for 50 minutes at $4{ }^{\circ} \mathrm{C}$. After discarding the

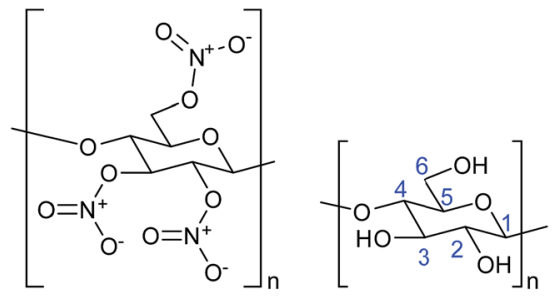

(a)

nitrocellulose

cellulose

(b)

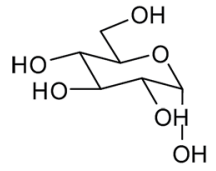

D-(+)-glucose

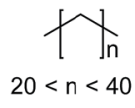

paraffin
Fig. 7 Molecular structures of the paper substrates (a) and filling substances (b).

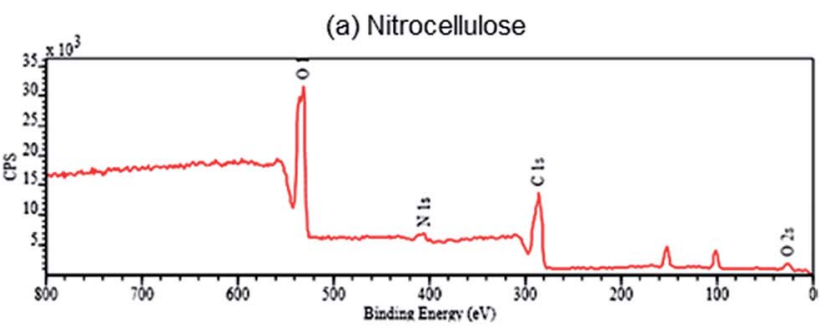

(b) Cellulose

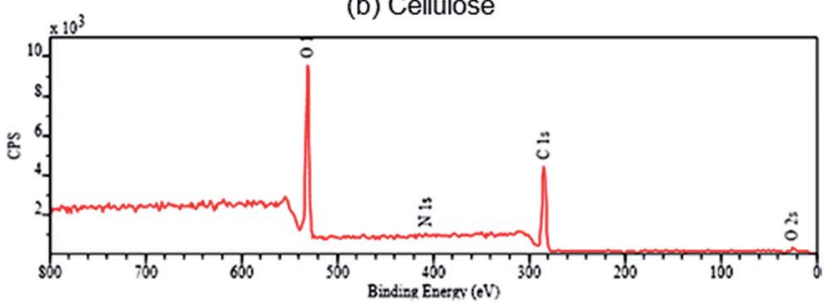

(c) Glucose-cellulose

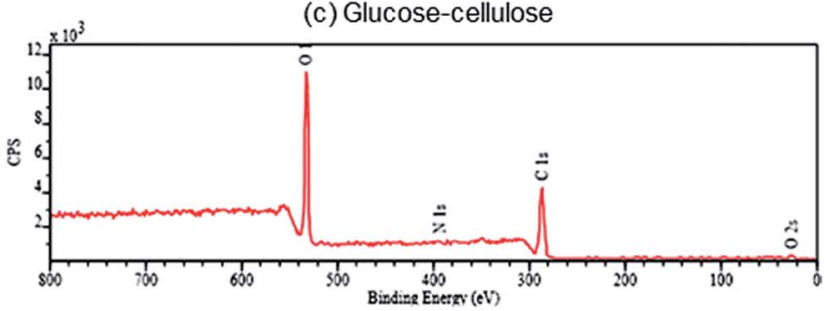

(d) Paraffin-cellulose

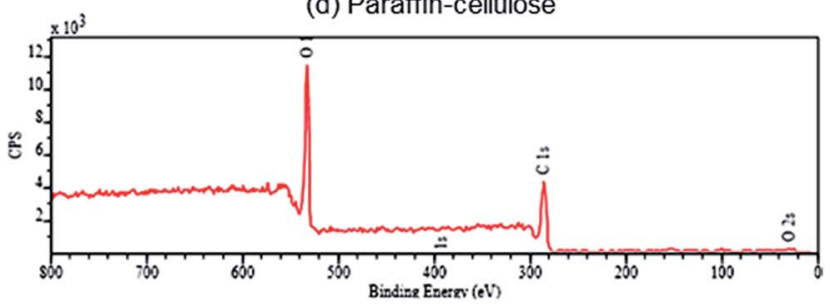

Fig. 8 XPS survey analysis of unprinted paper substrates. (a) is spectrum from nitrocellulose sheet, (b) from cellulose, (c) from glucosecellulose and (d) from paraffin-cellulose. The peaks corresponding to $\mathrm{O}$ 1s, $\mathrm{C}$ 1s and $\mathrm{N}$ 1s orbitals are labeled. 


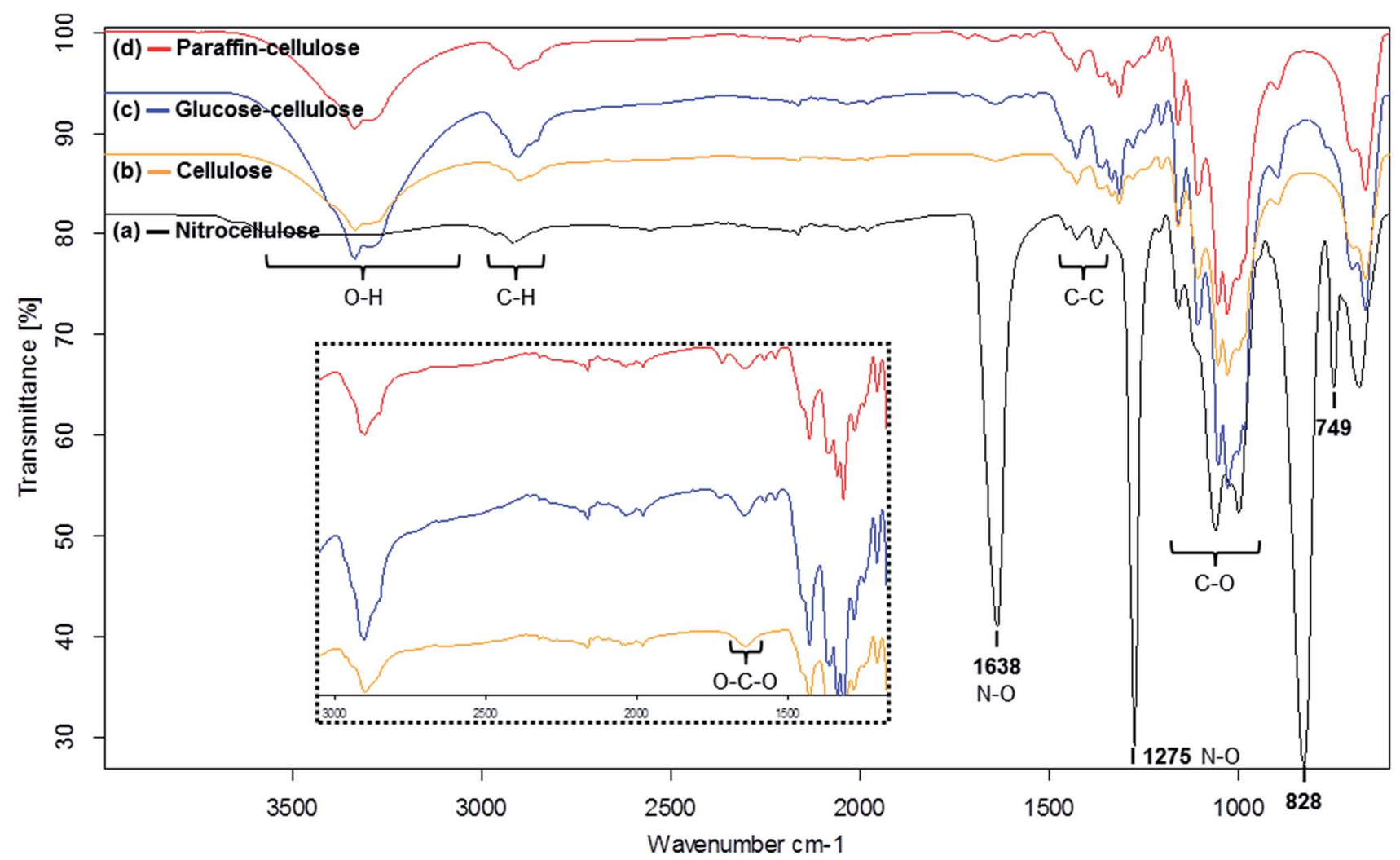

Fig. 9 IR spectra of unprinted paper substrates. (a) Is spectrum from nitrocellulose sheet, (b) from cellulose, (c) from glucose-cellulose and (d) from paraffin-cellulose. All spectra have several bands in common which correspond to $\mathrm{O}-\mathrm{H}, \mathrm{C}-\mathrm{H}, \mathrm{C}-\mathrm{C}, \mathrm{C}-\mathrm{O}$ and $\mathrm{O}-\mathrm{C}-\mathrm{O}$ stretching vibrations. The $\mathrm{N}-\mathrm{O}$ stretching vibrations specific to nitrocellulose are labeled.

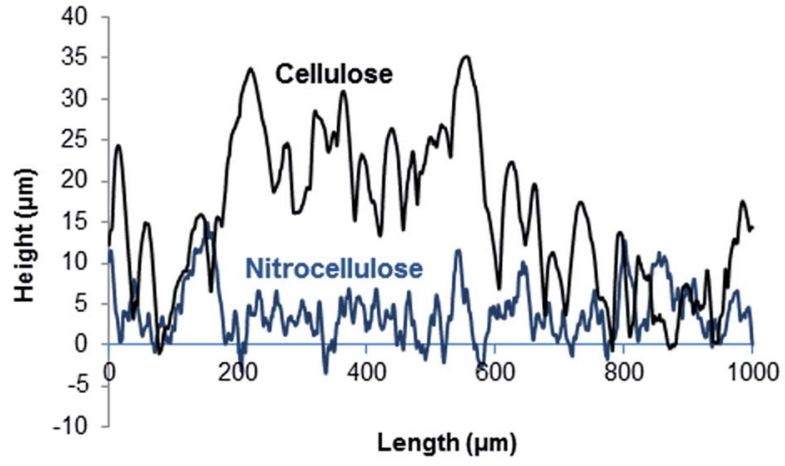

Fig. 10 Line profiles of the unprinted paper substrates.

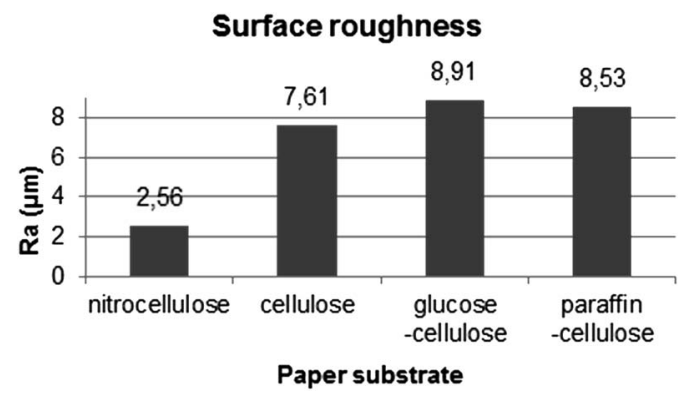

Fig. 11 Surface roughness $\left(R_{\mathrm{a}}\right)$ of the unprinted paper substrates. supernatant, the pellet was suspended in $250 \mu \mathrm{L}$ of $2 \mathrm{mM}$ borax buffer, $\mathrm{pH} 9.3$, containing $1 \%(\mathrm{w} / \mathrm{v}) \mathrm{BSA}$ and stored at $4{ }^{\circ} \mathrm{C}$ in the dark.

2.5.2. Preparation of immunochromatographic strips. An immunochromatographic strip is usually composed of a sample pad, a detection pad and an absorbent pad, the whole being affixed onto a plastic carrier (or backing card). Thus, an antibody-printed paper pad constituted the detection zone. In order to prevent nonspecific protein adsorption onto the detection membrane during immunoassays, all antibodyprinted membranes were saturated with a gelatin solution (0.1 M potassium phosphate buffer, $\mathrm{pH} 7.4$, containing $0.5 \%$ $(\mathrm{w} / \mathrm{v})$ porcine gelatin and $0.15 \mathrm{M} \mathrm{NaCl}$ ) overnight at $4{ }^{\circ} \mathrm{C}$, and then dried at $37^{\circ} \mathrm{C}$ in a ventilated oven for 30 minutes. All pads (about $20 \mathrm{~cm}$ width) were assembled onto the backing card and then the whole was cut into strips of $5 \mathrm{~mm}$ width (see Fig. 2).

2.5.3. Assessment of the immobilization. The test solution was composed of a goat anti-mouse tracer diluted 10 times in the analysis buffer. Unprinted parts of detection paper pads assessed the unspecific signal due to unspecific adsorption of the tracer onto the saturating matrix during immunoassays. The immobilization ability of the various paper substrates was therefore assessed by the colorimetric difference between the murine-antibody-printed part of detection pad (test line) and the unprinted corresponding one. 
(a) Nitrocellulose
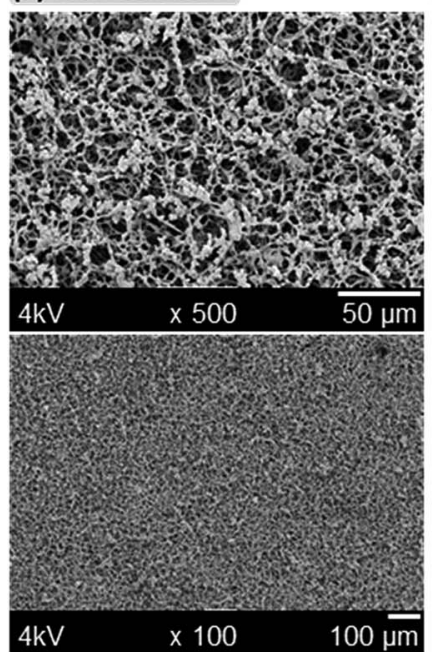

(b) Cellulose
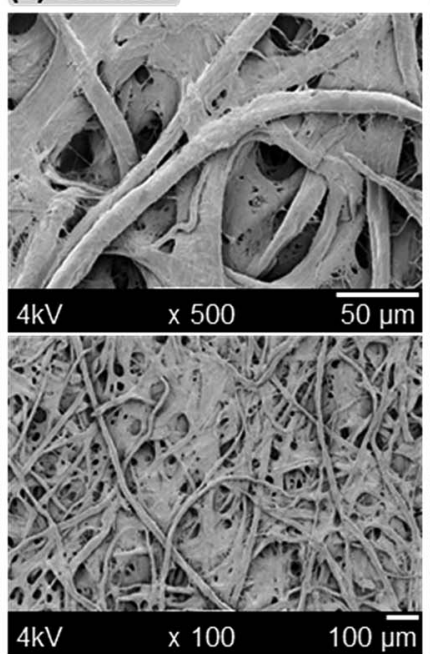

(c) Glucose-cellulose
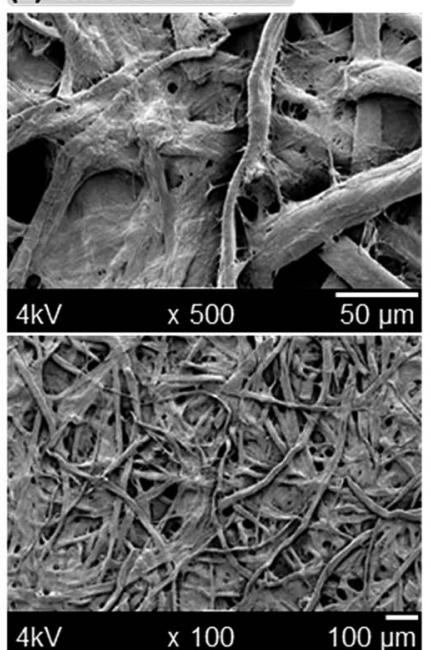

(d) Paraffin-cellulose
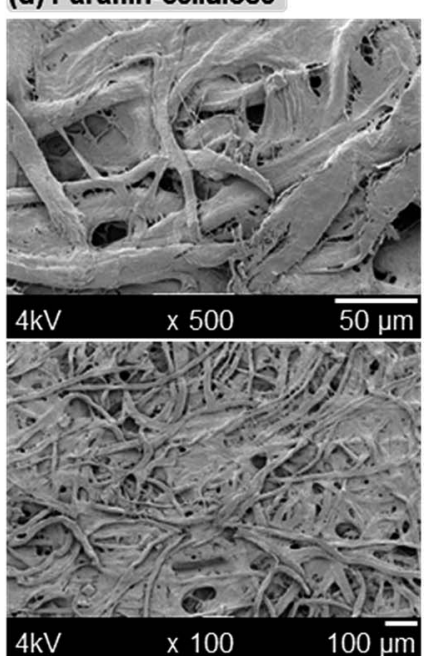

Fig. 12 SEM micrographs of unprinted nitrocellulose (a), cellulose (b), glucose-cellulose (c) and paraffin cellulose (d).

(a) Nitrocellulose

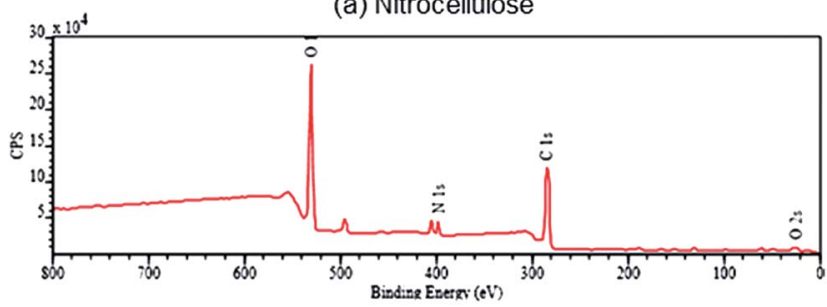

(b) Cellulose

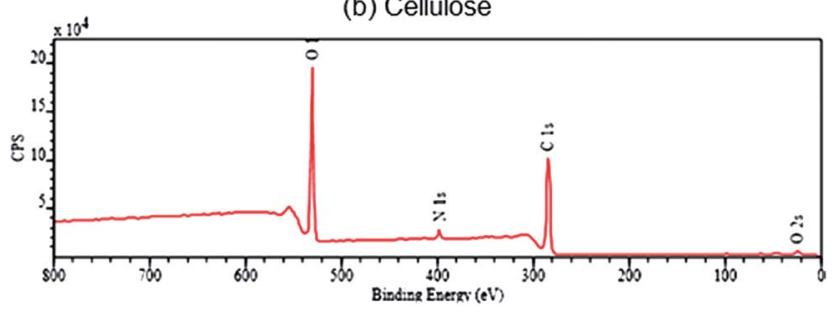

Fig. 13 XPS survey analysis of antibody-printed paper substrates. (a) is spectrum from nitrocellulose sheet, (b) from cellulose. The peaks corresponding to $\mathrm{O} 1 \mathrm{~s}, \mathrm{C} 1 \mathrm{~s}$ and $\mathrm{N}$ 1s orbitals are labeled.

2.5.4. Assessment of the biological activity and determination of the visual detection limit. Ten test solutions were prepared and pre-incubated for 15 minutes. The first one only contained murine anti-OVA mAb tracer diluted 10 times in the analysis buffer. This immunoassay without OVA antigen (0 ng $\mathrm{mL}^{-1}$ ) assessed the unspecific signal due to unspecific adsorption of the tracer onto the antibody-gelatin matrix during immunoassays (negative control). The nine others were solutions of murine anti-OVA mAb tracer (10-time dilution) and OVA (dilution series ranging from $1 \mathrm{ng} \mathrm{mL} \mathrm{m}^{-1}$ to $500 \mathrm{ng} \mathrm{mL}^{-1}$ ) in the analysis buffer.

The biological activity of the various paper substrates was therefore assessed by the colorimetric difference between the antibody-printed paper test-line signal in the presence of OVA and the corresponding one without OVA. Since it captured the excess murine anti-OVA tracer antibodies, the control line prevented false negative results. Its coloring guaranteed that the tracer actually passed through the test line, along with the test solution.

The visual detection limit (VDL) was determined through the OVA dilutions series. It was defined as the minimum OVA concentration resulting in a test-line colored signal significantly more intense than the negative control one.

\subsection{Patterned photoimmobilization of probe antibodies}

Probe antibodies, or colloidal-gold-labeled antibodies (tracers), were photoimmobilized onto pristine CF1 cellulose paper according to the following procedure. A $2 \mathrm{~cm}^{2}$ cellulose sheet $(2 \mathrm{~cm}$ $\times 1 \mathrm{~cm}$ in size) was manually impregnated with a goat anti-mouse tracer solution (3-fold dilution in the analysis buffer, $20 \mu \mathrm{L} \mathrm{cm}^{-2}$ deposit). Drying step was skipped and this system was then irradiated at $365 \mathrm{~nm}$ for $1 \mathrm{~h} 20 \mathrm{~min}$ (about $5 \mathrm{~J} \mathrm{~cm}^{-2}$ ) through an opaque plastic mask in order to localize the grafting (patterning process). Paper was rinsed overnight with the washing buffer. Colorimetric measurement using the molecular imager was performed immediately after the paper had been slightly dried over absorbent paper. The patterned image was pictured with either digital camera or VersaDoc ${ }^{\mathrm{TM}}$ Molecular Imager.

\section{Results and discussion}

\subsection{Localized immobilization of probe antibodies}

Photo-patterning consists in transferring an image displayed on a mask towards a substrate through photochemical or photoactivated reactions. This is the fastest and most easily undertaken process ensuring the localization of species onto a flat support according to a well-defined and reproducible pattern. This process was therefore combined to the photolinker-free photografting procedure previously patented $^{\mathbf{4 1 , 4 4}}$ in order to easily and rapidly localize antibodies onto cellulose sheets. 


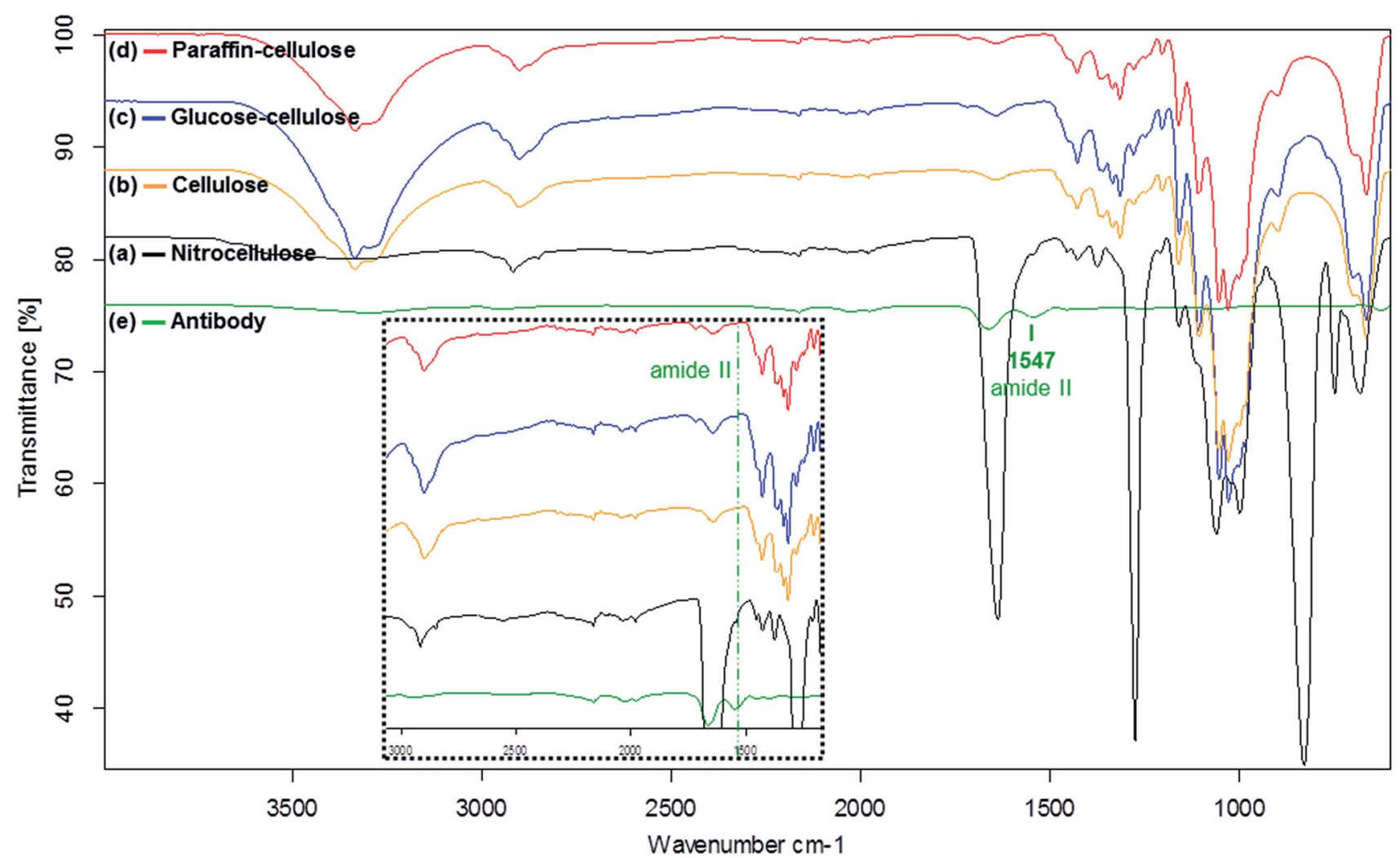

Fig. 14 IR spectra of antibody-printed paper substrates. (a) Is spectrum from nitrocellulose sheet, (b) from cellulose, (c) from glucose-cellulose and (d) from paraffin-cellulose. All spectra have several bands in common which correspond to $\mathrm{O}-\mathrm{H}, \mathrm{C}-\mathrm{H}, \mathrm{C}-\mathrm{C}, \mathrm{C}-\mathrm{O}$ and $\mathrm{O}-\mathrm{C}-\mathrm{O}$ stretching vibrations. The $\mathrm{N}-\mathrm{O}$ stretching vibrations specific to nitrocellulose are labeled.

Probe antibodies labeled with colloidal gold were immobilized through a mask in order to directly observe the photopatterned immobilization of antibodies, and to evaluate the signal/background ratio (Fig. 3). A selective photoimmobilization of the colloidal-gold-labeled antibody is observed according to the design of the used mask. This confirms the immobilization process to be photo-controlled.

The signal/background ratio is estimated to be around $140 \%$. Though it is a rather positive result, the high background colorimetric intensity also indicates that lots of antibodies are wasted in this process. That stems from the subtractive nature of the photo-patterning process. Thus, this process was set aside and an additive process such as inkjet printing was further preferred.

\subsection{From classical automatic dispensing to inkjet printing of antibodies}

Since automatic dispensing with BioDot-like systems ${ }^{46}$ is the most frequently used method for antibody dispensing onto immunoassay membranes, the inkjet printing approach was

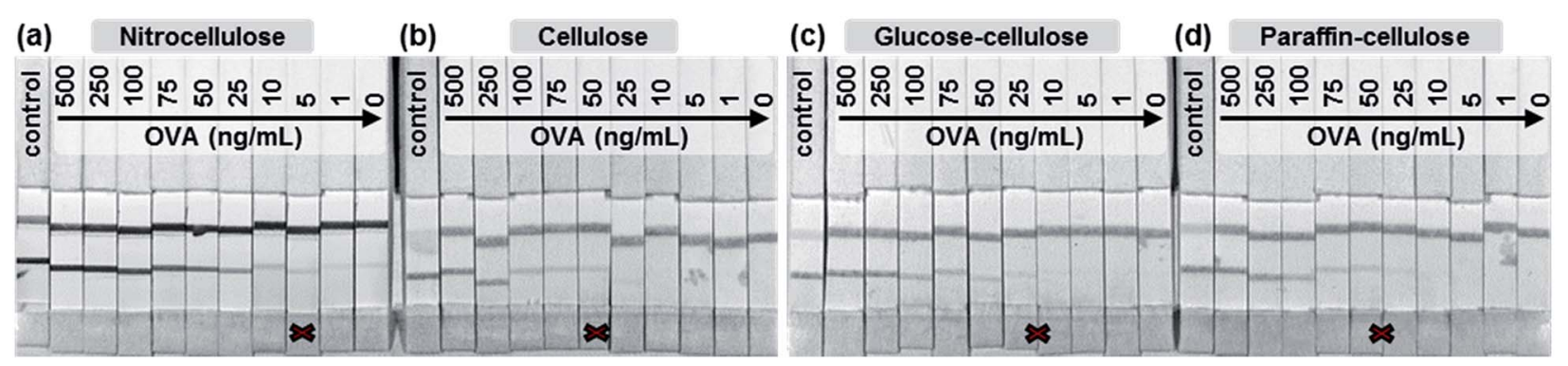

Fig. 15 Photographs showing the influence of the substrate and its pretreatment on biological activity and membrane VDL. The first set of strips (a) is made of nitrocellulose, the second (b) of cellulose, the third (c) of glucose-cellulose and the fourth (d) of paraffin-cellulose. Antibodies were adsorbed onto nitrocellulose and photoimmobilized onto cellulose-based substrates. Their actual immobilization was confirmed thanks to gold-labeled goat anti-mouse tracer (control strips). The capture of OVA antigen by the immobilized antibodies was highlighted by gold-labeled murine anti-OVA tracer (OVA strips). The strips corresponding to the membranes' VDL are labeled with a cross. Photographs were taken with the Molecular Imager. All experiments were reproduced 3 times but only one is shown here. 
(a)

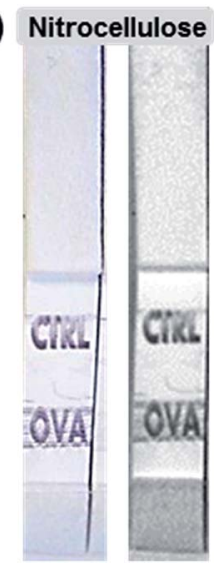

(b)

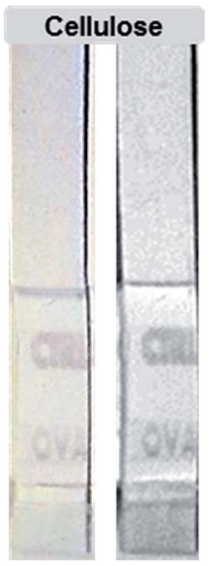

Fig. 16 Photographs showing the biological activity of antibodies printed according to a complex design. The first set of strips (a) was produced with nitrocellulose membrane, the second (b) with cellulose. Antibodies were adsorbed onto nitrocellulose and photoimmobilized onto cellulose. The capture of OVA antigen by the immobilized antibodies was highlighted by gold-labeled murine anti-OVA tracer. For each set of strips photographs were taken with both a digital camera (colored left pictures) and the Molecular Imager (grey right pictures).

first compared to the latter. That comparison aimed to validate the printing method for its use in the development of immunoassay devices.

Printings made of 1 and 5 layers were therefore compared to the single line deposit from the automatic dispenser (Fig. 4). After antibody solutions had been dispensed onto the substrates, the antibodies were either adsorbed onto nitrocellulose or photoimmobilized onto cellulose. First, their immobilization was confirmed by revelation with gold-labeled goat anti-mouse tracer (see control strips in Fig. 4). Then, their biological activity was put to the test by exposition to OVA antigen and simultaneously revealed by gold-labeled murine anti-OVA tracer (sandwich immunoassay) (see OVA strips in Fig. 4). Each test was performed in triplicate.

The first noticeable result is that the sets of strips obtained with BioDot dispensing method and with 5-layer inkjet printing are visually almost identical. Their coloring is quite strong, while the coloring resulting from 1-layer inkjet printing is obviously weaker. However, this weakness does not seem to lower its performances in terms of visual detection limit (VDL) as further detailed. This same set of strip actually displays slightly thinner and more precise test and control lines than the others, although they are all well-defined, thin and precise. With regard to biological activity, dilutive effect is clearly perceptible. Nevertheless, photographs reveal that the negative control (OVA at $0 \mathrm{ng} \mathrm{mL}^{-1}$ ) for nitrocellulose is slightly colored. This raises the issue of false positive results that can be observed with nitrocellulose immunoassay membranes. This issue does not arise with cellulose, most probably because of lower sensitivity. Considering that, the membranes' VDL were appraised as follows: (i) $5 \mathrm{ng} \mathrm{mL} \mathrm{mL}^{-1}$ for nitrocellulose and $25 \mathrm{ng}$ $\mathrm{mL}^{-1}$ for cellulose with BioDot dispensing method (Fig. 4a); (ii) 1 to $5 \mathrm{ng} \mathrm{mL}{ }^{-1}$ for nitrocellulose and $25 \mathrm{ng} \mathrm{mL}^{-1}$ for cellulose with 1-layer inkjet printing (Fig. 4b); and (iii) 1 to $5 \mathrm{ng} \mathrm{mL}^{-1}$ for

nitrocellulose and $25 \mathrm{ng} \mathrm{mL^{-1 }}$ for cellulose with 5-layer inkjet printing (Fig. 4c).

Each material VDL was therefore identical regardless the dispensing method or the number of layers. Thus, the printing process was indeed proved to be as efficient as the usual automatic dispensing, and therefore totally legitimate regarding its use in the development of immunoassay devices. Moreover, the printing method has the advantage of saving the quite expensive biomolecules dispensed because of the rather low ejected volume. Though an exact ejected volume could not be measured, the maximum dispensed volume was calculated based on the printer features (nominal drop volume, drop spacing and tension). For the selected pattern (a straight line of $600 \mu \mathrm{m}$ width), the printer was estimated to deliver $0.27 \mu \mathrm{L}$ $\mathrm{cm}^{-1}$ of antibody solution per layer. A maximum of $0.27 \mu \mathrm{L} \mathrm{cm}{ }^{1}$ of antibody solution was thus dispensed with 1-layer inkjet printing (Fig. 4b), a maximum of $1.35 \mu \mathrm{L} \mathrm{cm}^{-1}$ with 5-layer inkjet printing (Fig. 4c), and exactly $1 \mu \mathrm{L} \mathrm{cm}^{-1}$ with BioDot dispensing method (Fig. 4a). Since a 1-layer printing is efficient enough to determine the VDL, the consumed amount of antibodies is therefore nearly a quarter of the amount consumed with a classical automatic dispenser. Another advantage of printing over classical automatic dispensing is the freedom in design of the printed pattern (see further Section 3.4) while the usual automatic dispenser only allows drawing straight lines of rather undefined width.

Regarding the evaluation of the immobilization procedure, photoimmobilization onto cellulose led to VDL results in the same order of magnitude as the values obtained with adsorption onto nitrocellulose. However, cellulose performances appeared slightly lower than nitrocellulose's $\left(\mathrm{VDL}_{\text {cellulose }}=5\right.$ $\mathrm{VDL}_{\text {nitrocellulose}}$ ). Beyond procedure, this phenomenon might stem from the many differences both chemical and physical between the two substrates. This is why the experiments presented thereafter were dedicated to characterize these differences while trying to compensate for them by cellulose pretreatment.

\subsection{Inkjet printing of antibodies onto various substrates}

Beyond the obvious chemical difference in molecular structure, the main physical difference between nitrocellulose and cellulose substrates lies in their porosity (about $5 \mu \mathrm{m}$ and $11 \mu \mathrm{m}$ surface pore size, respectively) and sheet thickness (20 $\mu \mathrm{m}$ and $176 \mu \mathrm{m}$ thick, respectively). Since cellulose sheets with same porosity and thickness than nitrocellulose were not commercially available, cellulose pretreatments which aimed to compensate for that by filling cellulose pores were achieved. Given that the filling substance should be inert regarding antibody immobilization process and further immunoassays, two components were selected: glucose and paraffin. Glucose is the molecular repeating unit in cellulose macromolecule (see Fig. $7 a$ and $b)^{1}$ and therefore was not expected to disturb the immobilization process or further use of the membrane. In addition, its high water solubility $\left(180 \mathrm{mg} \mathrm{mL}^{-1}\right)$ would permit to easily remove it during post-irradiation washing step. Paraffin, a mixture of linear alkanes (see Fig. 7b), is well known 
for its unreactive nature. ${ }^{49}$ Unlike glucose, it is insoluble in water and therefore would stick into the fibers after the washing step and during further immunoassays.

Antibody solutions were printed onto the raw (nitrocellulose and cellulose) and pretreated (glucose-cellulose and paraffincellulose) substrates. Though 1 layer would have been enough, 5 layers were actually printed in order to get strong color intensity (see results Section 3.2). Antibodies were then adsorbed onto nitrocellulose substrate and photoimmobilized onto cellulose substrates (cellulose, glucose-cellulose and paraffin-cellulose). Surface morphological structure and chemical composition of both raw and pretreated substrates were analyzed prior to printing and afterwards. Printed antibody solutions were characterized as well. Finally, lateral flow immunoassays (LFIAs) ensured the ultimate characterization by evaluating the biological activity and visual detection limit of the various membranes.

$$
\dot{\gamma}=\frac{v}{h}
$$

$\dot{\gamma}$ is the shear rate $\left(\mathrm{s}^{-1}\right), v$ is the velocity $\left(\mathrm{m} \mathrm{s}^{-1}\right)$ and $h$ is the gap (m).

\subsubsection{Inks}

3.3.1.1. Composition. Printed solutions, also called inks, were antibody aqueous solutions. Because of different initial proportions in each antibody stock solution, their final salts content was different. Thus, murine anti-OVA antibody solution (test line ink) actually contained $1 \mathrm{mg} \mathrm{mL}^{-1}$ of monoclonal antibody (IgG) and $0.1 \mathrm{M}$ of potassium phosphate in water. Likewise, goat anti-mouse antibody solution (control line ink) contained $0.5 \mathrm{mg} \mathrm{mL}^{-1}$ of polyclonal antibody (IgG + IgM), 0.1 $\mathrm{M}$ of potassium phosphate and $0.05 \mathrm{M}$ of sodium chloride $(\mathrm{NaCl})$. These variations in salts content, but also in antibody type (IgG and IgM structures are depicted in Fig. 5 (ref. 50 and 51)) could greatly influence the surface tension between the antibody ink and the paper substrate, thereby inducing variations in the printing behavior.

3.3.1.2. Rheology. The viscosity of both test line and control line antibody solutions was measured (Fig. 6). As reminded in the previous section, test line ink consisted of murine anti-OVA monoclonal antibodies and control line ink of goat anti-mouse polyclonal antibodies. According to Fig. 6, control line ink viscosity varies from 2.28 to $1.69 \mathrm{mPa} \mathrm{s}$ when shear rate increases from 100 to $10000 \mathrm{~s}^{-1}$. A slight increase of viscosity is observed at shear rates higher than $2000 \mathrm{~s}^{-1}$. The control line solution is thus dilatant. Test line ink viscosity varies from 2.69 to $0.89 \mathrm{mPa} \mathrm{s}$ for the same shear rate ranges. The test line solution has a shear thinning behavior.

Eqn (1) is the expression of the shear rate as a function of gap and printing speed. When shear rate varies from 100 to 10000 $\mathrm{s}^{-1}$, speed varies from 0.01 to $1 \mathrm{~m} \mathrm{~s}^{-1}$ for a gap of $100 \mu \mathrm{m}(1 \times$ $\left.10^{-4} \mathrm{~m}\right)$. Depending on ink viscosity and printing voltage, jetting speed thus varies from 0.1 to $25 \mathrm{~m} \mathrm{~s}^{-1} \cdot{ }^{52,53}$ Hence, high shear rates larger than $10000 \mathrm{~s}^{-1}$ and exceeding the rheometer measuring limits may be estimated.

Ideally, an inkjet printing ink must be Newtonian with a constant viscosity (1-10 $\mathrm{mPa}$ ) at varying shear rates. ${ }^{54}$ Though not Newtonian, biomolecule solutions are inkjet printable because of their low viscosities $(<2 \mathrm{mPa} s)$.

\subsubsection{Initial substrates}

3.3.2.1. Molecular structure. Cellulose is a natural biopolymer made up of glucose units (Fig. 7a). It is the simplest polysaccharide since it is composed of a unique monomer (glucose) which binds to its neighbors by a unique type of linkage ( $\beta-1,4$ glycosidic bond resulting in acetal function). ${ }^{1}$ According to its molecular structure, hydroxyl groups in glucose units are responsible for cellulose chemical activity. ${ }^{55}$ However, this group cannot directly interact with proteins, what makes cellulose activation or functionalization necessary in order to covalently bind to proteins of interest.

Cellulose pretreatments introduced few additive molecules but did not change the native molecular structure of cellulose. Additive substances were adsorbed onto it and partially filled its pores. These additives were glucose and paraffin. While glucose is the molecular repeating unit in cellulose macromolecule, paraffin is a mixture of linear alkanes (see Fig. 7b).

Nitrocellulose (also named cellulose nitrate) is the most important cellulose derivative. Biomolecules strongly adsorb to nitrocellulose through a combination of electrostatic, hydrogen, and hydrophobic interactions involving the nitro functions. ${ }^{45}$ It is therefore the reference material for performing lateral flow immunoassay (LFIA). ${ }^{\mathbf{1 0 , 1 1 , 4 5}}$ Cellulose nitrate is formed by esterification of hydroxyl groups from cellulose (primary or secondary) with nitric acid in the presence of sulfuric acid, phosphoric acid or acetic acid (see Fig. 7a). ${ }^{55,56}$

These molecular features represent the first, but not most, difference between the nitrocellulose and cellulose-based substrates.

3.3.2.2. Surface chemical analysis. The outer surface layers of paper substrates were analyzed by surface chemical analysis such as XPS and ATR-FTIR, thereby displaying the aforementioned bulk molecular structures.

XPS allows the identification of elements within $10 \mathrm{~nm}$ deep subsurface layers. ${ }^{48}$ All papers are mainly composed of carbon and oxygen and therefore the XPS signal for these two elements is quite strong on every spectrum shown. Fig. 8 displays $O 1 \mathrm{~s}$ orbital binding energy at $532 \mathrm{eV} \pm 0.35 \mathrm{eV}, \mathrm{O} 2 \mathrm{~s}$ orbital binding energy at $24 \mathrm{eV} \pm 0.35 \mathrm{eV}$ and $\mathrm{C} 1 \mathrm{~s}$ orbital binding energy at 284 $\mathrm{eV} \pm 0.35 \mathrm{eV}) .{ }^{48}$ Another peak at $405 \pm 0.35 \mathrm{eV}$ is noticeable onto nitrocellulose spectrum which is attributable to $\mathrm{N} 1 \mathrm{~s}$ orbital.

According to its layout, ATR-FTIR allows the identification of chemical bonds within $2 \mu \mathrm{m}$ deep subsurface layers. ${ }^{57}$ All papers are mainly composed of a cellulosic backbone and therefore the IR signals for its typical bond vibrations are shared by every spectrum shown. Fig. 9 displays these common bands attributable to $\mathrm{O}-\mathrm{H}, \mathrm{C}-\mathrm{H}, \mathrm{C}-\mathrm{C}, \mathrm{C}-\mathrm{O}$ and $\mathrm{O}-\mathrm{C}-\mathrm{O}$ stretching vibrations. Besides, nitrocellulose manifests additional peaks (1638 \pm 5 $\mathrm{cm}^{-1}$ and $1275 \pm 5 \mathrm{~cm}^{-1}$ ) attributable to $\mathrm{N}-\mathrm{O}$ stretching vibrations.

3.3.2.3. Surface morphological structure. Beyond the chemical differences in molecular structure, the main difference between nitrocellulose and cellulose substrates lies in their surface physical structure. Thus, topological analysis was conducted in order to quantify the surface morphological structure 
by measuring its roughness $\left(R_{\mathrm{a}}\right)$. SEM imaging allowed visualizing surface morphology and microstructure of the unprinted substrates.

Line profiles of unprinted paper substrates (Fig. 10) reveal that nitrocellulose surface is more homogeneous, smoother and has fewer and narrower pores compared to cellulose-based paper surfaces. Since profiles of the three cellulose-based papers were quite similar, only cellulose profile is displayed on Fig. 10. Surface roughness $\left(R_{\mathrm{a}}\right)$ values (Fig. 11) confirm that nitrocellulose is way smoother than cellulose-based papers. Pores size and arrangement pictured by SEM imaging (Fig. 12) also corroborate the previous statements. SEM micrographs and roughness profiles predict that with the same ejected volume of antibodies, thicker and better resolution patterns will be printed on nitrocellulose. Thus, lower visual detection limits are expected to be reached with nitrocellulose membranes. This was supported by Määttänen et al. ${ }^{58}$ who demonstrated that wetting rate reduces with surface roughness increase. Besides, they explained that ink is quickly and completely absorbed into the depth of porous surfaces, thus leaving less ink deposit onto the substrate surface.

According to SEM imaging (Fig. 12), glucose treatment seems to barely affect cellulose surface aspect. On the other hand, when paraffin treatment was performed, fewer pores were observed onto the surface. Regarding surface roughness (Fig. 11), an increase was displayed by both glucose and paraffin treatments.

\subsubsection{Printed substrates}

3.3.3.1. Surface chemical analysis. After antibody had been printed onto the various paper substrates, their outer surface layers were analyzed anew in order to detect any change stemming from the biomolecules. The XPS signal from carbon and oxygen is still quite strong on every spectrum shown (Fig. 13). Additional peaks at $397.5 \pm 0.35 \mathrm{eV}$ have come out onto all the spectra which are attributable to $\mathrm{N}$ 1s orbital from antibody molecules. Since spectra of the three cellulose-based papers were quite similar, only cellulose spectrum is displayed on Fig. 13.

With regard to IR analysis, the intense spectra from initial substrates hid most of the characteristic bands pointing out the immobilized antibodies (Fig. 14). Therefore, the amide bands specific to proteins are barely perceivable. Only amide II at 1547 $\pm 5 \mathrm{~cm}^{-1}$ could be clearly identified onto nitrocellulose substrates.

3.3.3.2. Surface morphological structure. After antibody had been printed onto the various paper substrates, their surface morphology and microstructure were visualized anew (not shown) by SEM imaging in order to detect any change stemming from the biomolecules. Unfortunately, the microscope resolution was not high enough to enable a direct visualization of antibody deposit. However, a thin new layer seems to have appeared on cellulose-based substrates when comparing to Fig. 12.

3.3.4. Lateral flow immunoassays (LFIAs). Antibody solutions were printed onto the raw (nitrocellulose and cellulose) and pretreated (glucose-cellulose and paraffin-cellulose) substrates. 5-layers were printed in order to get strong color intensity. Antibodies were then adsorbed onto nitrocellulose substrate and photoimmobilized onto cellulose substrates (cellulose, glucose-cellulose and paraffin-cellulose). Lateral flow immunoassays (LFIAs) evaluated the biological activity of the printed antibodies and the visual detection limit of the various bioactive membranes, thereby allowing characterization of the various substrates in terms of biosensing performances. First, the immobilization ability of the various membranes was confirmed by revelation with gold-labeled goat anti-mouse tracer (see control strips in Fig. 15). Then, their biological activity was assessed by exposition to OVA antigen and revealed by gold-labeled murine anti-OVA tracer (sandwich immunoassay) (see OVA strips in Fig. 15). Each test was performed in triplicate.

The first fact to notice is that though antibodies were barely perceivable with the various surface analysis performed (XPS, IR or SEM), they are well visible after either revelation with goat anti-mouse tracer (control strips) or bioactivity assessing immuno sandwich (OVA strips). With regard to biological activity, few aforementioned results (see Section 3.2) remain. Dilutive effect is still clearly perceptible. There is still a false positive result with nitrocellulose that compels to appraise its

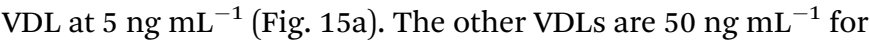
cellulose (Fig. 15b), 10 to $25 \mathrm{ng} \mathrm{mL}^{-1}$ for glucose-cellulose (Fig. 15c), and 25 to $50 \mathrm{ng} \mathrm{mL}{ }^{-1}$ for paraffin cellulose (Fig. 15d). While nitrocellulose's VDL is still the same as in Section 3.2, cellulose's VDL is now higher. Since all test lines coloring seems weaker than in Fig. 4c, this inter-assay variability could originate from tracer variability due to the use of another batch of colloidal gold. On another hand, the intra-assay comparison of the different substrates reveals that both glucose and paraffin enrichment only slightly improved cellulose performances although they are still lower than nitrocellulose's. Besides, glucose-cellulose appeared to be the most sensitive cellulosebased substrate. This could be explained by a slight decrease in surface porosity, as expected; though this decrease was not really significant regarding nitrocellulose porosity. But this most probably stemmed from the preservative and stabilizing effect of glucose on biomolecules. ${ }^{59}$

\subsection{Inkjet printing of complex designs}

As previously mentioned, one advantage of inkjet printing dispensing method is the freedom in design of the printed pattern. This advantage was illustrated here by printing antibodies according to their nature and function, thereby making the user manual not so useful anymore. Since bottom line was dedicated to capture OVA antigen, murine anti-OVA monoclonal antibodies printing drew the abbreviation OVA. Similarly, anti-mouse antibodies were printed on the top line according CTRL abbreviation as the top line aimed to control the smooth progress of the immunoassay. After antibody solutions had been dispensed onto the substrates (1-layer inkjet printing), the antibodies were either adsorbed onto nitrocellulose or photoimmobilized onto cellulose. Their biological activity was put to the test by exposition to OVA antigen (500 $\mathrm{ng} \mathrm{mL}^{-1}$ ) and simultaneously revealed by gold-labeled 
murine anti-OVA tracer (Fig. 16). Colors observed, along with their intensities, were consistent with previous results (see Section 3.2). Finally, as expected, the drawn patterns allowed direct reading of the test results. This process therefore enables to doubly check the nature of the target antigen (on the box and on the strip), thereby avoiding ambiguousness when box label is partly erased. Firstly, this can permit to save valuable assay devices in remote areas in the developing world. In addition, this double-check can be a huge asset in developed countries in emergency situations, in emergency rooms or in military settings, where the result of the assay impacts on people's lives.

\section{Conclusion}

A fast, simple, cost-saving and environmentally friendly process for strong and precisely localized immobilization of antibodies onto paper has been described herein. This new approach combines inkjet printing of biomolecules with a photolinkerfree photografting procedure which together enable to easily, rapidly and permanently immobilize antibodies onto cellulosebased papers according to any pattern desired. The inkjet printing dispensing method has the great advantage of saving the expensive biomolecules. The photografting procedure has the one of being harmless to chemical-sensitive biomolecules. The process was first tested in the development of simple lateral flow immunoassay (LFIA) device and then applied to more complex LFIA devices. Membranes' performances were evaluated in terms of visual detection limit (VDL). Several parameters of the process have been studied (printing parameters, cellulose pretreatment), hence resulting in membranes challenging nitrocellulose performances. Cellulose performances appeared slightly lower than nitrocellulose's though. But this phenomenon probably stemmed from the physical differences, such as surface porosity variation, between nitrocellulose and cellulose substrates.

This research was carried out to meet need for paper-based sensing device development to rapidly, robustly and abundantly immobilize biomolecules onto cellulose sheets according to complex patterns and at low cost. Meanwhile, the first part of the process developed - the inkjet printing dispensing method by itself - also proved itself to be efficient and useful with nitrocellulose reference material. More generally, the expounded process provides a powerful tool for immobilizing chemical-sensitive proteins according to complex patterns and onto various cellulose-based paper sheets.

\section{Acknowledgements}

This work was financially supported by the Commissariat à l'Energie Atomique et aux Energies Alternatives (France). Jocelyne Leroy is greatly acknowledged for performing the XPS analysis and helping in analyzing XPS data. Dr Rocío de Miguel Viscasillas and Marine Gay are greatly acknowledged for lending the Sputter Coater and helping in using it.

\section{Notes and references}

1 J. Credou and T. Berthelot, J. Mater. Chem. B, 2014, 2, 47674788.

2 D. Klemm, B. Heublein, H.-P. Fink and A. Bohn, Angew. Chem., Int. Ed., 2005, 44, 3358-3393.

3 R. L. Crawford, Lignin biodegradation and transformation, John Wiley \& Sons Inc, New York, NY, USA, 1981.

4 S. Kalia, B. S. Kaith and I. Kaur, Cellulose Fibers: Bio- and Nano-Polymer Composites, Springer, Berlin, Heidelberg, 2011.

5 E. J. Maxwell, A. D. Mazzeo and G. M. Whitesides, MRS Bull., 2013, 38, 309-314.

6 A. W. Martinez, S. T. Phillips, G. M. Whitesides and E. Carrilho, Anal. Chem., 2010, 82, 3-10.

7 J. Credou, H. Volland, J. Dano and T. Berthelot, J. Mater. Chem. B, 2013, 1, 3277-3286.

8 Materials Research Society, MRS Bull., 2013, 38, 294-352.

9 R. Hawkes, E. Niday and J. Gordon, Anal. Biochem., 1982, 119, 142-147.

10 G. A. Posthuma-Trumpie, J. Korf and A. van Amerongen, Anal. Bioanal. Chem., 2009, 393, 569-582.

11 B. Ngom, Y. Guo, X. Wang and D. Bi, Anal. Bioanal. Chem., 2010, 397, 1113-1135.

12 J. Burstein and G. D. Braunstein, Early Pregnancy: Biol. Med., 1995, 1, 288-296.

13 T. Chard, Hum. Reprod., 1992, 7, 701-710.

14 A. W. Martinez, S. T. Phillips and G. M. Whitesides, Proc. Natl. Acad. Sci. U. S. A., 2008, 105, 19606-19611.

15 SENTINEL: Bioactive Paper Network, http:// www.bioactivepaper.ca/index.php? module $=$ page $\&$ id $=4000$, (accessed Jan 31, 2014).

16 S. Aikio, S. Grönqvist, L. Hakola, E. Hurme, S. Jussila, O.-V. Kaukoniemi, H. Kopola, M. Känsäkoski, M. Leinonen, S. Lippo, R. Mahlberg, S. Peltonen, P. Qvintus-Leino, T. Rajamäki, A.-C. Ritschkoff, M. Smolander, J. Vartiainen, L. Viikari, and M. Vilkman, Bioactive paper and fibre products: Patent and literary survey, Oulu, Finland, 2006.

17 E. M. Fenton, M. R. Mascarenas, G. P. López and S. S. Sibbett, ACS Appl. Mater. Interfaces, 2009, 1, 124-129.

18 X. Li, D. R. Ballerini and W. Shen, Biomicrofluidics, 2012, 6, 011301.

19 P. Lisowski and P. K. Zarzycki, Chromatographia, 2013, 76, 1201-1214.

20 L. Ge, S. Wang, X. Song, S. Ge and J. Yu, Lab Chip, 2012, 12, 3150-3158.

21 D. D. Liana, B. Raguse, J. J. Gooding and E. Chow, Sensors, 2012, 12, 11505-11526.

22 E. Carrilho, S. T. Phillips, S. J. Vella, A. W. Martinez and G. M. Whitesides, Anal. Chem., 2009, 81, 5990-5998.

23 E. Carrilho, A. W. Martinez and G. M. Whitesides, Anal. Chem., 2009, 81, 7091-7095.

24 S. Wang, L. Ge, X. Song, J. Yu, S. Ge, J. Huang and F. Zeng, Biosens. Bioelectron., 2012, 31, 212-218.

25 T. Songjaroen, W. Dungchai, O. Chailapakul and W. Laiwattanapaisal, Talanta, 2011, 85, 2587-2593. 
26 A. Määttänen, U. Vanamo, P. Ihalainen, P. Pulkkinen, H. Tenhu, J. Bobacka and J. Peltonen, Sens. Actuators, B, 2012, 177, 153-162.

27 A. V. Govindarajan, S. Ramachandran, G. D. Vigil, P. Yager and K. F. Böhringer, Lab Chip, 2012, 12, 174-181.

28 L. Lafleur, D. Stevens, K. McKenzie, S. Ramachandran, P. Spicar-Mihalic, M. Singhal, A. Arjyal, J. Osborn, P. Kauffman, P. Yager and B. Lutz, Lab Chip, 2012, 12, 1119-1127.

29 E. Grinenval, G. Nonglaton and F. Vinet, Appl. Surf. Sci., 2014, 289, 571-580.

30 A. Douvas, P. Argitis, K. Misiakos, D. Dimotikali, P. S. Petrou and S. E. Kakabakos, Biosens. Bioelectron., 2002, 17, 269-278.

31 P. S. Petrou, M. Chatzichristidi, A. M. Douvas, P. Argitis, K. Misiakos and S. E. Kakabakos, Biosens. Bioelectron., 2007, 22, 1994-2002.

32 D. J. Graber, T. J. Zieziulewicz, D. a. Lawrence, W. Shain and J. N. Turner, Langmuir, 2003, 19, 5431-5434.

33 S. M. Zakir Hossain, R. E. Luckham, A. M. Smith, J. M. Lebert, L. M. Davies, R. H. Pelton, C. D. M. Filipe and J. D. Brennan, Anal. Chem., 2009, 81, 5474-5483.

34 K. Abe, K. Kotera, K. Suzuki and D. Citterio, Anal. Bioanal. Chem., 2010, 398, 885-893.

35 X. Li, J. Tian, G. Garnier and W. Shen, Colloids Surf., B, 2010, 76, 564-570.

36 D. Gamota, P. Brazis, K. Kalyanasundaram and J. Zhang, Printed Organic and Molecular Electronics, Springer, USA, Boston, MA, 2004.

37 J. Birkenshaw, Printed Electronics (Pira on printing), Leatherhead, Surrey, UK, 2004.

38 K. Abe, K. Suzuki and D. Citterio, Anal. Chem., 2008, 80, 6928-6934.

39 P. Jarujamrus, J. Tian, X. Li, A. Siripinyanond, J. Shiowatana and W. Shen, Analyst, 2012, 137, 2205-2210.

40 F. Kong and Y. F. Hu, Anal. Bioanal. Chem., 2012, 403, 7-13. 41 J. Credou and T. Berthelot, EP14157944, 2014.

42 J. Credou, R. Faddoul, and T. Berthelot, EP14179401.6, 2014.

43 J. Credou, R. Faddoul, and T. Berthelot, EP14180423.7, 2014.

44 J. Credou, H. Volland and T. Berthelot, J. Mater. Chem. B, 2015, 3, 1079-1088.
45 R. C. Wong and H. Y. Tse, Lateral Flow Immunoassay, Humana Press, New York, NY, 2009.

46 N. Khreich, P. Lamourette, H. Boutal, K. Devilliers, C. Créminon and H. Volland, Anal. Biochem., 2008, 377, 182-188.

47 N. Khreich, P. Lamourette, P.-Y. Renard, G. Clavé, F. Fenaille, C. Créminon and H. Volland, Toxicon, 2009, 53, 551-559.

48 L.-S. Johansson and J. M. Campbell, Surf. Interface Anal., 2004, 36, 1018-1022.

49 H. Noh and S. T. Phillips, Anal. Chem., 2010, 82, 4181-4187.

50 G. T. Hermanson, Bioconjugate techniques, Academic Press, London, 2008.

51 A. Makky, T. Berthelot, C. Feraudet-Tarisse, H. Volland, P. Viel and J. Polesel-Maris, Sens. Actuators, B, 2012, 162, 269-277.

52 Fujifilm. Dimatix Materials Printer DMP-2831, http:// www.fujifilmusa.com/products/ industrial_inkjet_printheads/deposition-products/dmp2800/\#overview, (accessed Jun 4, 2014).

53 A. Denneulin, J. Bras, F. Carcone, C. Neuman and A. Blayo, Carbon, 2011, 49, 2603-2614.

54 A. Blayo and B. Pineaux, in Proceedings of the 2005 joint conference on Smart objects and ambient intelligence innovative context-aware services: usages and technologies sOc-EUSAI'05, ACM Press, New York, NY, USA, 2005, pp. 27-30.

55 D. Roy, M. Semsarilar, J. T. Guthrie and S. Perrier, Chem. Soc. Rev., 2009, 38, 2046-2064.

56 D. Klemm, B. Philipp, T. Heinze, U. Heinze, and W. Wagenknecht, Comprehensive Cellulose Chemistry Volume 2 Functionalization of Cellulose, WILEY-VCH, Weinheim, vol. 2, 1998.

57 PIKE Technologies, MIRacle ATR; Product Data Sheet, Madison, WI, USA, 2014.

58 A. Määttänen, P. Ihalainen, R. Bollström, M. Toivakka and J. Peltonen, Colloids Surf., A, 2010, 367, 76-84.

59 D. Y. Stevens, C. R. Petri, J. L. Osborn, P. Spicar-Mihalic, K. G. McKenzie and P. Yager, Lab Chip, 2008, 8, 2038-2045. 